Aus dem I. anat. Institut in Wien.

\title{
Über den Einfluß der Kastration auf den Organismus.
}

\author{
III. "Die Eunuchoide.k
}

Von

Julius Tandler und Siegfried Grosz.

Mit Tafel VIII-X.

Eingegangen am 17. Februar 1910.

Wenn wir in unsrer zweiten Mitteilung ${ }^{1}$ ) die Einflußnahme der Keimdrüse auf den menschlichen Organismus durch extreme Ausfallserscheinungen dartun konnten, so wollen wir nunmehr jene Veränderungen beschreiben, welche sich aus der Unterentwicklung der Geschlechtsdrtise und der daraus folgenden Unterfunktion ergeben.

Hierhergehörige Einzelbeobachtungen finden sich in der Literatur unter verschiedenen Bezeichnungen vielfach angeführt. Auch die Abhängigkeit bestimmter somatischer Erscheinungen von der Unterentwicklung der Keimdrise wurde schon des öfteren hervorgehoben. Eine systematische Untersuchung des ganzen Komplexes an der Hand eines größeren Materials steht bis heute aus. Eine solche erscheint um so erwünschter, als die Symptomatologie eine mannigfaltige ist, demgemäß verschiedene Autoren das eine oder andre Symptom in den Vordergrund rïcken und nach diesem das Gesamtbild benennen. Dementsprechend sehen wir auch eine verwirrende Fülle in der Nomenclatur. Wenn wir für den ganzen Komplex der Äußerungen, welche sich im Gefolge von Genitalhypoplasie ergeben, den Ausdruck »Eunuchoid * wählen, so veranlassen uns hierzu folgende Gründe.

Erstens, daß sie die allgemeinste ist, zweitens, daß sie die Ahnlichkeit mit dem Eunuchentypus betont. Diese Bezeichnung wurde unsres Wissens zunächst von englischen Autoren - GRIFFITHS und

1) Die zweite Mitteilung, die Skopzen betreffend, erscheint im 30. Bande dieses Archivs (Festschrift für W. Roux). 
DucKWoRTH - gebraucht, in der deutschen Literatur hat sie sich bisher nicht einzubürgern vermocht. Die sonst gebrauchten Bezeichnungen, wie Dysgenitalismus, infantilisme avec gigantisme, Dystrophia adiposo-genitalis, l'obésité d'origine génitale, Geroderma genitodistrofico usw., stellen ein bestimmtes, besonders hervorstechendes Symptom in den Vordergrund; die betreffenden Einzelbeobachtungen stellen nur Typen jener großen Gruppe von Krankheitserscheinungen dar, die wir unter der Bezeichnung "Eunuchoide zusammenfassen wollen. So wie wir bei Eunuchen zwei Typen unterschieden haben, nämlich den mit Disproportion im Längenwachstum und den mit excessiver Fettanhäufung, so wollen wir auch bei den Eunuchoiden diese zwei Haupttypen, nämlich den eunuchoiden Hochwuchs bzw. die eunuchoide Disproportion und den eunuchoiden Fettw uchs unterscheiden.

Wenn wir nun darangehen, einzelne unsrer bezüglichen Beobachtungen zur Mitteilung zu bringen, sei bemerkt, daß diese nur Beispiele aus einer viel größeren $Z$ ahl von Fällen darstellen. Wir haben nur jene Fälle ausgewählt, welche als Paradigmen Interesse verdienen, oder bei denen wir, dank der günstigen äußeren Umstände, photographische Aufnahmen machen konnten. Wir wollen betonen, daß es sich beim Eunuchoid keineswegs um Seltenheiten handelt, daß man vielmehr den Vertretern dieser Entwicklungsanomalie sehr häufig begegnet, da sie einen eigenartigen Typus darstellen. An dieser Stelle müssen wir den Chefs der beiden chirurgischen Kliniken in Wien, den Herren Hofräten v. Eiselsberg und Hochenegg, ferner Herrn Hofrat Palitauf und den Herren Doz. Dr. Ewald und MaResch und Dr. RoBinson unsern wärmsten Dank aussprechen.

An die Spitze der von uns zu veröffentlichenden Fälle stellen wir einen, bei welchem der Obduktionsbefund und die histologische Untersuchung beigebracht werden können.

\section{I.}

Am 27. November 1908 kam ein etwa 28jähriger Mann, M. H., mit Lungentuberkulose zur Sektion (Prosektor Dozent Dr. MARESCH). Der erhobene Befund lautet auszugsweise:

Kopfhaar dicht, vor beiden Ohren spurenweise kurze Lanugohärchen, ebenso am Kinn und an der Oberlippe, sonst rollkommen bartlos. Axillarhaare spärlich, wenige an der Peniswurzel befindliche Schamhaare. Trotz der Magerkeit der Leiche relativ gut entwickelter Fettwulst in der Unterbauchgegend. 


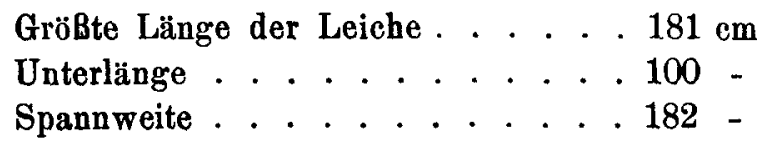

Penis auffallend klein, Skrotum kurz, in demselben zwei kleine Testikel. Beim Betasten läßt sich eine unverhältnismäßig große Epididymis gegen den kleinen Testikel abgrenzen. Prominentia laryngea fehlt, der Schildknorpel deutlich federnd. Da uns einige Tage später die ganze Leiche zur Verfugung gestellt wurde, haben wir sie skeletiert, da die Weichteile für eine mikroskopische Untersuchung bereits unverwendbar waren. Am Orte der Sektion waren das Genitale and der Kehlkopf in Formalin konserviert worden.

Wir wollen zunächst das Skelet beschreiben. An demselben fällt die Grazilität und die Länge der Röhrenknochen auf, ferner das Offenbleiben bestimmter Epiphysenfugen. Die Höhe des Skelets beträgt $175 \mathrm{~cm}$. Die Spannweite $180 \mathrm{~cm}$.

$$
\begin{aligned}
& \text { Unterlänge mißt . . . . } 98 \mathrm{~cm} \\
& \text { Oberlänge - . . . } 77 \text { - } \\
& \text { Schädelmaße: } \\
& \text { Umfang . . . . . . } 50 \mathrm{~cm} \\
& \text { Schädellänge . . . . . 15,8 - } \\
& \text { Schädelbreite . . . . . 13,4 - } \\
& \text { kleinste Stimbreite . . . } 9 \text { - } \\
& \text { Ohrhöhe . . . 9,6 - } \\
& \text { Gesichtshöhe . . . . 10,2 - } \\
& \text { Jochbreite . . . . . . 11,5 - }
\end{aligned}
$$

Der Gehirnschädel ist schwach asymmetrisch, die Sutura sagittalis ist bereits einfach gezackt, während in der Coronarnaht und in den Parieto-occipitalnähten noch tertiäre Nahtzackung nachweisbar ist.

An der Innenfläche des Schädels sind die Sulci arteriosi und venosi sowie die Impressiones digitatae sehr tief. Die Foveae cerebellares sind gut entwickelt. Die Sella turcica ist beilänfig $11 \mathrm{~mm}$ lang und etwa $16 \mathrm{~mm}$ breit, $11 \mathrm{~mm}$ tief, vom Tuberculum sellae bis auf die Basis der Hypophysengrube gemessen. Die Hypophysengrube selbst ist deutlich vertieft.

Der Margo supraciliaris ist gut entwickelt.

Die Nasenwurzel ist tief gesattelt, die Nasenbeine sind breit, die Jochbeine plump. Die Protuberantia mentalis ist flach, der Unterkieferwinkel ist auffällig groB, der Unterkieferkörper gegenuber 
Über den Einfuß der Kastration auf den Organismus. III.

dem sonst grazilen Schädel verhältnismäßig dick. An den Schneidezähnen des Ober- und Unterkiefers Schmelzdefekte, in Form von Querfurchen.

\section{BeckenmaBe:}

Dist. spin. il. ant. snp. . . . . . . . . $22 \mathrm{~cm}$

Dist. spin. il. post. sup.. . . . . . . . 6,8 -

Dist. crist. . . . . . . . . . . 25,5 -

Länge der Crista rechts. . . . . . . 26 -

- - - links . . . . . . 25,5 -

Breite des Sacrum am Beckeneingang . 10 -

Länge des Sacrum . . . . . . . . 13 -

Diameter transv. . . . . . . . . . 12 -

Conjugata vera. . . . . . . . . 10,7 -

Diameter obliq. rechts . . . . . . 12,5 -

- links. . . . . . . 12,2 -

Distant. der Spin. ischiad.. . . . . . . 9,3 -

Entfernung der Kreuzbeinspitze von der Spin. ischiad. . . . . . . . . . 5,4 -

Höhe des Foramen obtur. . . . . . . . 5 -

Breite - - - . . . . . 3,5 -

Größte Höhe des Beckenkanals, gemessen

vom Tubercul. ileopect. zum Tuber ossis

isch. . . . . . . . . . 10,5 -

Obere Extremität:

GröBte Länge des Humerus links . . . . $34 \mathrm{~cm}$

- $\quad-$ der Ulna links . . . . . . 27 -

- $\quad$ - des Radius links . . . . 251/2 -

Untere Extremität:

GröBte Länge des Femur . . . . . . $53 \mathrm{~cm}$

$$
\begin{aligned}
& \text { - } \quad-\text { der Tibia . . . . . . . 40 - } \\
& \text { - - - Fibula ...... 39,5- - }
\end{aligned}
$$

Offene Epiphysenfugen finden sich am sternalen Claviculaende, spurenweise am acromialen. Vollkommen offen sind die Epiphysenfugen am proximalen Humerusende, am distalen Ende des Radius und der Ulna. Ferner am distalen Ende des Femur und am proximalen Ende der Tibia und der Fibula. Schließlich befinden sich Epiphysen an der Crista ilei und am Tuber ossis ischii (Fig. 1). 
Von den Eingeweiden standen uns das Genitale und der Kehlkopf zur Verfügung. Am Kehlkopf fehlt die Verknöcherung der Kehlkopfknorpel vollständig. Seine Dimensionen erinnern an die des Kindes, Prominentia laryngea fehlt, der Winkel, den die beiden Laminae thyreoideae einschließen, ist groß. An der Glandula thyreoidea ist nichts Auffälliges vorhanden.

Ganz auffällig ist zunächst die geringe Entwicklung des Hodens (Fig. 3). Dieser mißt $13 \mathrm{~mm}$ in seiner größten Ausdehnung, während er zusammen mit dem Nebenhoden gemessen $27 \mathrm{~mm}$ Längsdurchmesser hat. Dementsprechend ist die Epididymis ganz unverhältnismäßig stark entwickelt im Vergleich mit dem minimalen Hoden. Am Vas deferens fehlt die ampulläre Erweiterung, die Samenbläschen sind klein, wenig verzweigt. Die Prostata (Fig. 2) ist ebenfalls klein, derb und gegen die Nachbarschaft wenig abgesetzt. Der Penis ist klein, die Urethra mißt im ganzen $11,5 \mathrm{~cm}$, die Corpora cavernosa penis sind sehr klein, das Corpus, cavernosum urethrae dagegen besser entwickelt.

Die mikroskopische Untersuchung des Genitales ergibt folgendes:

Hoden. Die Tunica albuginea des Hodens ist sehr dick. Die spärlichen Hodenkanälchen zeigen ein vielgestaltiges Bild insofern, als sich bei der Durchmusterung der Schnitte fast alle Stadien der mangelhaften Ausbildung derselben nachweisen lassen. An den noch am besten entwickelten Kanälchen sieht man eine dicke, ringförmige, hyalin aussehende Tunica propria. Das Lumen ist erfillt von Zellen, deren Protoplasmaleib und Kern gut färbbar ist. Eine Verschiedenheit zwischen den einzelnen Zellen, aus welcher man eine Differenzierung derselben erschließen könnte, fehlt vollkommen. Diese Kanälchen gleichen bis zu einem gewissen Grade den Hodenkanälchen von Kindern. An andern Kanälchen sieht man die Zellen weniger gut gefärbt, protoplasmaarm, brïcken- und strangförmig das Lumen unterteilend. An vielen Kanälchen mit besonders kräftiger hyaliner Tunica sind nur mehr einzelne Zellen nachweisbar, bis schließlich auch diese schwinden und solide hyaline Stränge zurückbleiben.

Das zwischen den Hodenkanälchen befindliche Gewebe ist verhältnismäßig reich an elastischen Fasern, lockerem Bindegewebe, und zeigt einzelne Zellverbände, deren Zellen in Form and Größe und der Art ihres Verbandes den Zwischenzellen gleichen. Von normalen Zwischenzellen unterscheiden sie sich durch ihre auffällig geringe Färbbarkeit, durch ihre Armut an Protoplasma, durch ihr mehr hyalines Aussehen. 
Wie viel von diesen Abweichungen von den normalen Zwischenzellen als durch die nicht einwandfreie Konservierung bedingt $\mathrm{zu}$ betrachten ist, läßt sich nicht bemessen. Die Blutgefäße sind zahlreich, dickwandig.

Nebenhoden. Wie schon bei der makroskopischen Beschreibung erwähnt, ist der Nebenhoden im Vergleich zu dem Hoden unverhältnismäBig gut entwickelt. Das mikroskopische Querschnittsbild der Epididymis zeigt auch insofern normale Verhältnisse, als die Weite der einzelnen Kanälchen sowie ihre Zahl eine annähernd normale ist. Sowohl der Ductus deferens als auch die Ductuli efferentes zeigen ein weites Lumen, das stellenweise von einer fein retikulierten, wenig färbbaren Masse erfullt ist. Wie am normalen Organ lassen sich zwei Reihen von Zellen unterscheiden; die periphere kubische Basalzellenreihe, einer dtunen, geschichteten Lamina propria aufsitzend, ist normal, während die innere Cylinderzellenreihe besonders protoplasmaarm ist, stellenweise einen scharfen Lumensaum anfweist, hingegen nur spärliche sekretorische Vorgänge erkennen läßt. Die bindegewebig-muskuläre Hülle der einzelnen Kanälchen ist am Querschnitt schmäler als normal, anßerdem scheinbar muskelärmer und weniger dentlich gegen das interstitielle Bindegewebe abgrenzbar.

Prostata. Die Prostata gleicht der seinerzeit von uns beim Eunuchen beschriebenen fast vollständig. Anch hier fällt zunächst die Armut an Drusen auf, wenn sie auch keine so hochgradige ist wie beim Eunuchen. Die einzelnen Drüsenquerschnitte sind unverhältnismäßig weit, von einem zweizeiligen, relativ hohen Epithel ausgekleidet. Nur an wenigen Stellen sieht man lebhaft Eosin annehmende, schollige, vielfach auch konzentrische Schichtung aufweisende Sekretballen. Das bindegewebig-muskuläre Stroma der Vorsteherdrüse ist verhältnismäßig gut entwickelt. Der Querschnitt der beiden Ductus ejaculat. innerhalb der Drüse zeigt ein relativ weites Lumen, das von einem niedrigen Epithelsaum ausgekleidet ist und von scholligen, keinerlei Struktur aufweisenden, stark eosingefärbten Massen erfuillt ist.

II.

Adolf L., 50 Jahre alt (Fig. 4). Patient ist verheiratet, Vater eines etwa 13jährigen Kindes, gibt an, daB er sexuell niemals besonders leistungsfähig gewesen sei. Während aber früher angeblich Erektionen eintraten, sollen diese in den letzten Jahren immer seltener geworden sein. 


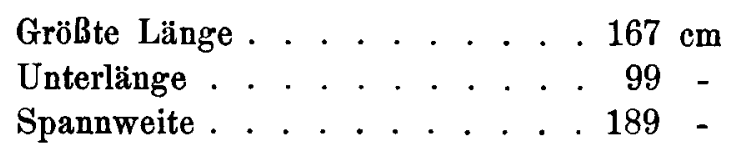

Kopfhaar reichlich, angeblich früh ergraut.

Die Haut des Gesichts mit Furchen und Falten versehen, blaß, vollkommen bartlos. Einige feine Lanugohärchen finden sich an der Oberlippe. An den gut ausgebildeten Augenbrauen fehlen die stärkeren längeren Borstenhaare, welche dem Alter des Individuums entsprechend vorhanden sein müßten.

Am Hals, der fettreich ist, fehlt die Prominentia laryngea. Die Cartilago thyreoidea weist keine Zeichen vorgeschrittener Verknöcherung auf. Die Gl. thyreoidea ist nicht tastbar. Kastratenstimme.

Der Stamm ist vollkommen haarlos. Das subkutane Fettgewebe ist hier gut entwickelt. Axillarhaare sehr spärlich. Die Mammae prominent und fettreich. In der Unterbauchregion nimmt das Fett stark $\mathrm{zu}$ und grenzt sich gegen den fettreichen Mons veneris durch eine tiefe Querfurche ab. Diese Querfurche steigt beiderseits, allmählich flacher werdend, gegen die Spina anterior superior auf, um sich daselbst zu verlieren. Diese Konfiguration der seitlichen unteren Bauchgegend erinnert an die bei Kindern vorkommenden Verhältnisse.

Am Mons veneris sind nur wenige lange Crines pubis vorhanden, das behaarte Feld begrenzt sich nach oben horizontal. Der Penis ist klein, das Skrotum schlaff, völlig haarlos. Die Testikel haselnußgroß, gegen den besser entwickelten Nebenhoden deutlich abgrenzbar.

Das Perineum ist vollkommen haarlos.

An den Nates und an den rỉckwärtigen Teilen der Cristae iliacae ist das Fett stark entwickelt.

Die Beckenneigung ist eine geringe.

Genu valgum beiderseits.

III.

Karl K., 51 Jahre alt. (Fig. 5 u. 6).

Patient, welcher schwer arbeitet, gibt an, sich vollkommen gesund zu fuhlen. Er behauptet, den Beischlaf niemals ausgefuhrt zu haben, da er keinerlei Neigung znm weiblichen Geschlecht empfinde.

Es handelt sich um einen mittelgroßen, fetten, anffällig hochbeinigen Mann. haare.

Kopfhaar dicht, gran; Angenbrauen gat entwickelt, ohne Borsten- 
Das Gesicht fahl, mit vielen Furchen und Falten versehen, vollkommen bartlos.

Prominentia laryngea fehlt; Kastratenstimme.

Fettanhäufung in der Region der Mammae, ferner in der Unterbauchregion in besonderer Mächtigkeit, an den Cristae iliacae und ad nates.

Spärliche Axillarhaare, Crines pubis wenig entwickelt.

Penis sehr klein, Testikel kaum erbsengroß.

Epiphysenfugen geschlossen, eine Vergrößerung der Sella turcica nicht nachweisbar.

IV.

Heinrich Str., 26 Jahre alt (Fig. 7).

Patient gibt an, niemals Geschlechtstrieb empfunden zu haben. Es handelt sich um ein relativ fettes, hochbeiniges Individuum.

Kopfhaar, Augenbrauen normal entwickelt.

Keine Spur von Barthaaren, Prominentia laryngea fehlt.

Axillarhaare spärlich, ebenso Crines pubis, welche sich gegen die Unterbauchregion mit einer horizontalen Linie begrenzen.

Mächtige Fettansammlung an den Mammae, in der Unterbauchregion, an den Cristae iliacae und ad nates.

Penis klein, Testikel kaum erbsengroß.

Am proximalen Humerusende Epiphysenfugen nicht mehr nachweisbar. Am Röntgenbilde erscheint die Sella turcica von normaler Größe.

V.

Josef Sch., 25 Jahre alt (Fig. 8).

Angeblich seit der Geburt fett; gibt an, niemals Geschlechtstrieb empfunden zu haben.

Patient ist $162 \mathrm{~cm}$ groB, auffällig fett. Speziell in der Unterbauchgegend kam es zur Entwicklung eines tuberhängenden Fettpolsters, so daß Patient die chirurgische Entfernung desselben verlangte. Es wurde ihm an der Klinik v. EISELSBERg ein etwa $6 \mathrm{~kg}$ schweres Fettstiick aus der Unterbauchregion exstirpiert.

Die Rumpfmaße sind folgende:

Umfang in der Brustwarzenhöhe . . . . . . $101 \mathrm{~cm}$

- - - Nabelhöhe . . . . . . . . 106 -

- zwischen Nabel und Symphyse . . . . 108 -

Zwei Querfinger oberhalb der Symphyse. . . . . 110 - 
Kopfhaar und Augenbrauen normal entwickelt. Gesicht sehr fettreich, vollkommen bartlos. Axillar- und Schambare fehlen vollständig.

Genitale infantil, Testikel erbsengroß.

Genu valgum. Kastratenstimme.

Epiphysenfugen sämtlich offen.

Keine nachweisbare Vergrößerung der Sella turcica.

VI.

Hans E., 16 Jahre alt, $170 \mathrm{~cm}$ lang, $85 \mathrm{~kg}$ schwer, nachdem er kurz vorher eine Entfettungskur durchgemacht hat. Sehr fett.

Die Haut des Gesichts zart, ohne Spur von Bart.

Prominentia laryngea fehlt, der Knabe hat noch nicht mutiert.

Spärliche Axillarhaare, einzelne wenige Crines pubis. Mächtige Fettentwicklung an den Mammae, die frauenhaft roluminös sind. Starke Fettentwicklung in der Unterbauchregion, an den Cristae und an den Nates.

Penis sehr klein, der eine Hoden nicht auffindbar, der andre bohnengroß.

Genu valgum beiderseits geringgradig entwickelt.

Am Roentgenbilde die Sella turcica scheinbar etwas verbreitert.

An den Metacarpalknochen die Epiphysenfugen noch offen.

VII.

E. B., 14 Jahre alt (Fig. 9).

Vater 43 Jahre alt, ist in den letzten Jahren erst dick geworden, zुibt an, wenig zu essen. Schwestern des Vaters schon in jungen Jahren sehr fett, sind sämtlich unverheiratet.

Es sind im ganzen funf Kinder, die Mädchen sollen chlorotisch sein, ebenso die Schwestern der Mutter an Chlorose gelitten baben.

Maße: Gesamtlänge . . . . . . . . $157 \mathrm{~cm}$

Spannweite. . . . . . . . 164,5 -

Die Stirn ist auffällig breit, der Margo supraorbitalis flach und plump. Die Augenbrauen sind lateralwärts stark verbreitert, die Haare der Schläfenregion gehen in die seitlichen Augenbrauen direkt uber. Die Nasenwarzel ist gut gesattelt, Stumpfnase. Große Interorbitaldistanz. Auf der Oberlippe finden sich lichte und einzelne dunkle Lanugohaare, an Wangen und Kinn lichte. Die untere Kinnregion ist sehr fett, der Gaumen sehr hoch gewölbt. 
Am fettreichen Halse sind die Seitenlappen der Thyreoidea undeatlich, der Isthmus gut tastbar.

An der Vertebra prominens ein Fetthöcker, mächtiger Panniculus adiposus am Sternum, ebenso Mammae sebr fettreich. Der Umbilicus ist stark eingezogen.

Das Fett der Unterbauchregion tiber handbreit dick, durch eine deatliche Furche gegen die fette Regio pubis abgeschlossen. Inguinalfalten infantil, angedeutetes Genu valgum.

Starke Fettentwicklung am Darmbeinkamm und am Oberschenkel.

Genitale: Penis kurz, Skrotum faltig, lebhafter Cremasterreflex vorhanden.

Testikel sind anfänglich in der fetten Inguinalregion völlig verborgen, lassen sich schließlich nach abwärts verschieben, hängen an einem straffen Samenstrang.

Hoden und Nebenhoden lassen sich voneinander separieren, sind relativ hart. Rechter Hoden: Querdurchmesser etwa $1 \mathrm{~cm}$, Längsdurchmesser etwa $11 / 2 \mathrm{~cm}$. Linker Hoden spurenweise kleiner, weniger konsistent.

\section{VIII.}

K., 12 Jahre alt, sehr fett.

An der Oberlippe spärliche Lanugohaare, auffallend große Mammae, dicke, fettreiche Oberarme, keine Axillarhaare, mächtige Entwicklung des Fettes in der Unterbauchgegend, starke Fettpolster an den Hüften und Nates.

Penis von der Größe des eines fünfjährigen Knaben, Testikel bohnengroß.

Vater des Knäben gibt an, daß derselbe bezüglich seines Sexus sehr kindlich sei, daß sich keine Zeichen einer beginnenden Pubertät zeigen.

$$
\begin{aligned}
& \text { Länge . . . . . . . . . } 152 \mathrm{~cm} \\
& \text { Unterlänge . . . . . . . . . . } 79 \text { - }
\end{aligned}
$$

Ein Jahr später ergibt sich bei der Besichtigung desselben Knaben folgender Befund: Das Gewicht hat trotz der Größenzunahme eine Verringerung erfahren, die Lanugohare der Oberlippe haben zugenommen, Axillarhaare fehlen, spärliche Crines pubis. Bedeutende Zunahme der Hodengröße.

Der genau beobachtende, äußerst intelligente Vater gibtan, daß sich der Charakter des Knaben auffällig geändert habe. Er ist mitteilsamer geworden, selbstän- 
diger, und hat einen Teil seines kindlichen Gehabens verloren.

IX.

H., 12 Jahre alt, $71 \mathrm{~kg}$ schwer (Fig. 10).

Es handelt sich um einen auffällig dicken Knaben, der wegen Osteomyelitis des rechten Oberschenkels in chirurgischer Behandlung der Klinik Hochenegg stand.

Axillarhaare, Crines pubis fehlen.

Genitale hypoplastisch, Penis sehr klein, Testikel kaum erbsengroß.

Mächtige Fettanhäufungen an den Mammae, an der Unterbauchregion, an den Cristae iliacae und ad nates.

Genu valgum geringen Grades.

Außerdem bringen wir noch eine kleine Zahl von Photographien, Fälle betreffend, welche bezuglich Anamnese, Körpermaße sich typisch verhalten, so daß es uns überflüssig erscheint, eine genauere Wiedergabe der betreffenden Daten zu bringen. Um so eher, als die charakteristischen Photogramme für sich selbst sprechen.

Fig. 11 betrifft einen 43 jährigen Mann, A. E.

Fig. 12 betrifft einen 20 jährigen Mann, St.

Fig. 13 betrifft einen Fall H., der von Schüller in der Gesellschaft für innere Medizin vorgestellt wurde (1909).

Wie schon oben bemerkt, haben wir das hier veröffentlichte Material so zu gruppieren versucht, daß wir jene zwei Typen unterschieden, welche sich auch bei der Beschreibung der Kastraten ergeben haben:

1) eunuchoider Hochwuchs bzw. ennuchoide Disproportion,

2) eunuchoider Fettwuchs.

Naturgemäß stehen diese beiden Typen nicht scharf begrenzt einander gegentiber, sondern es lassen sich eine Reihe von Übergangsformen insofern nachweisen, als einzelne Charaktere den Vertretern beider Typen gemeinschaftlich sind, weiter, als einzelne Merkmale bei dem einen oder andern Typus mehr hervortreten oder verwischt sind.

ad 1) Was den von uns aufgestellten ersten Typus betrifft, erhebt sich die Schwierigkeit, im Einzelfalle zu bestimmen, ob ein Individnum schon als »hochwtichsig* zu bezeichnen ist. Die Gesamtlänge kann za einer solchen Entscheidung nur bedingungsweise herangezogen 
werden. Dazu wäre die Kenntnis jener Größe notwendig, welche das Individuum unter normalen Verhältnissen erreicht hätte.

So berechtigt uns nur der Umstand, daß die Kastraten ibre normalen Stammesgenossen durchschnittlich an Länge übertreffen im Zusammenhalt mit den gleichsinnigen Ergebnissen der Tierkastration zu der Aussage, daß dieses gesteigerte Längenwachstum durch den Wegfall der Keimdrüse bedingt ist.

Auch die von uns beobachteten Fälle von eunuchoidem Hochwuch s übertreffen die durchschnittliche Länge normaler Personen der hiesigen Bevölkerung. Charakteristischer für den Skeletbau solcher Individuen ist die Disproportion desselben, welche sich folgendermaßen änßert: gesteigertes Längenwachstum der Extremitäten, demgemäß lange Arme, lange Beine. Die besondere Armlänge ist nachweisbar durch das $\mathrm{Maß}$ der Klafterweite, die Beinlänge durch das Überwiegen der Unterlänge gegenüber der Oberlänge.

Dabei trägt das Skelet als Kennzeichen der Unreife offene Epiphysenfugen jahrelang uber den Zeitpunkt hinaus, zu welchem sie an normalen Personen zu verschwinden pflegen.

Die bei der Beschreibung des Eunuchenskelets hervorgehobenen Charakteristika, wie Sattelnase, veränderte Beckenform, Genu valgum u. a. finden sich auch hier in mehr oder weniger ausgeprägter Form, wie vor allem aus der Beschreibung des zur Obduktion gelangten Eunuchoiden hervorgeht.

In andern Fällen ist die Gesamtlänge des Individuums trotz des gesteigerten Wachstums eine das Durchschnittsmaß nicht übertreffende, ja sie kann unter diesem bleiben. Dann verbleibt als Kennzeichen der Zugehörigkeit zur Gruppe des » eunuchoiden Hochwachses « nur die eben geschilderte Disproportion. Eben diese ist es auch, welche den eunuchoiden Typus vom infantilen trennt, sofern man unter Infantilismus das Erhaltenbleiben der kindlichen Körperproportionen versteht.

Diese Begriffsabgrenzung erscheint deshalb von Belang, weil sie von manchen Autoren bei der Benennung einschlägiger Krankheitsbilder nicht hinlänglich berïcksichtigt wurde. So wird beispielsweise der Infantilismus, Typus Lorrain, geschildert als » par des individus de petite taille mais dont les segments sont proportionés comme ceux des adultes ${ }^{1}$ ). On ne trouve pas de disproportion entre la tête et le trone ....

1) So verstand Lask̀gue unter Infantilismus eine Hemmungsbildung, welche Archiv f. Entwicklungsmechanik. IXIX. 
Aus einem ähnlichen Grunde erscheint uns auch die Benennung -infantilisme arec gigantisme , die auch von deutschen Autoren verwendet wurde, keine gliuckliche.

ad 2) Was den Skeletbau der zu diesem Typus gehörenden Individuen betrifft, so ist die Disproportion wohl vorhanden, doch weniger ausgeprägt, die länger persistierenden Epiphysenfugen, sowie die das übrige Skelet betreffenden Veränderungen (Becken, Genu valgum usw.) sind auch hier anzutreffen.

Charakteristisch für den eunuchoiden Fettwuchs ist die Form und Lokalisation des Fettansatzes. So wie beim analogen Kastratentypus findet sich auch hier der Fettansatz an den oberen Augenlidern, an den Mammae (Gynaekomastie) ${ }^{1}$ ), Unterbauchregion, Cristae iliacae und Nates.

Haben wir somit die Merkmale aufgezählt, durch welche die beiden von uns aufgestellten Typen charakterisiert sind, so wollen

sich charakterisiert durch die Fortdauer der physischen und seelischen Merkmale der Kindheit. Lorrain gab folgende Definition:

1) Debilität, Grazilität und abnormes Kleinbleiben des Körpers, eine Ent wicklungsstörung, welche mehr die Gesamtmasse des Körpers, als irgend ein spezielles Organ betrifft.

2) Fortdauer der Jugendmerkmale, so daß ein Mann mit 30 Jahren etwa wie ein Achtzehnjähriger erseheint.

3) Außerdem rechnete LoRRain auch die feminine Abartung hierher.

Axton, Die Formen und die Ursachen des Infantilismus. Deutscher Verein für Psychiatrie. Ztsehr. f. Psych. Bd. 63.

Anton defininiert den allgemeinen Infantilismus als seine Entwicklungsstörung, welche den ganzen Organismus auf kindlichem Typus zurïckbleiben läßt und die Fortentwicklung des Individuums im Sinne seiner Gattung verhindert; dabei bleiben nicht nur die körperlichen Merkmale, sondern vielfach auch die seelischen Eigenschaften des Kindes fortbestehen*.

ScHüLLER unterscheidet den sdystrophischen Infantilismus and den durch Blutdrüsenanomalien bedingten Infantilismus, drittens den idiopathischen Infantilismus oder primären Dysgenitalismus. Hierher gehören die Fälle von infantilem Riesenwuchs mit atrophischen und funktionsuntuichtigen Keimdrüsen, mangelhaften sekundären Geschlechtscharakteren, übermäßigem Skeletwachstum, mangelhafter geistiger Entwicklung, weiter jene Fälle von Infantilismus, welche durch eine Kombination von Hypoplasie der Keimdrïsen mit Adipositas und Imbezillitït charakterisiert sind. *

1) $>$ Die Gynaekomastie ist eine Anomalie, welche in der übermäßigen und dauernden Entwicklung der Brüste beim Manne im Moment der Pubertät $\mathrm{zu}$ gleich unter Hemmung der Entwicklung der Hoden besteht.:

E. Laurent, >Die Zwitterbildungen. Gynaekomastie, Feminismus, Hermaphrodismus. Übersetzt von S. H. KureLLA. Leipzig 1896. 
wir nunmehr jene besprechen, welche ihnen gemeinsam sind und sie als $\gg$ Eunuchoide" kennzeichnen.

Dahin gehören vor allem die Veränderungen der Haut. Die Haut des Gesichts ist bei jugendlichen Individuen auffällig zart, blaß; schon frühzeitig gewinnt sie jedoch durch die Ausprägnng zahlloser Falten und Runzeln ein greisenhaftes Aussehen und zeigt ein fahlgelbes Kolorit. Dieser Beschaffenheit entsprechend haben einzelne Autoren dem ganzen Symptomenkomplex die Bezeichnung Geroderma gegeben. Dabei sind das Haupthaar und die Angenbrauenhaare dicht, auf das Ausbleiben der Borstenhaare, wie sie de norma im späteren Mannesalter in den Augenbrauen aufzutreten pflegen, haben wir bereits hingewiesen. Die Haut des Gesichts bleibt vollkommen bartlos, nur im höheren Alter sieht man einzelne Haare am Kinn, am Übergange der Oberlippe in die Wange auftreten, ein Behaarungstypus, wie er sich häufig bei alten Frauen findet und den wir auch bei alten Kastraten beschreiben konnten.

Die Haut des Stammes und der Extremitäten ist vermöge der Fettunterpolsterung zart, blaß, dabei haarlos; die bei Männern sonst vorkommende Behaarung an der Brust, an den Schenkeln, an den Vorderarmen und Händen fehlt. Axillarhaare sind nur spärlich vorbanden, die Behaarung der Regio pubis ist gleichfalls eine spärliche, schließt nach oben geradlinig ab. Bei weiblichen Personen bleiben die Haare auf die Labia majora beschränkt, so daß der Mons veneris fast vollkommen haarfrei ist. Das Perineum ist ebenfalls unbehaart.

Das Kehlkopfskelet bleibt, wie das des Kastraten, bis in das späte Alter knorpelig. Die beiden Laminae thyreoideae schließen einen großen Winkel ein, die Prominentia laryngea fehlt, der Kehlkopf erinnert in seinen Dimensionen an den kinälichen. Die betreffenden Individuen haben eine hohe, unausgeglichene Stimme.

Betreffs der Schilddrüse sei bemerkt, daß wir dieselbe in unsern Fällen niemals vergrößert fanden, in der Regel war sie schwer oder gar nicht tastbar.

Die Thymus ist, soweit unsre Erfahrung reicht, länger persistent als normal. So konnten wir an den Röntgenogrammen einen der Thymus entsprechenden Schatten nachweisen, ähnlich wie bei Kastraten.

Der Penis ist auffallig klein, die Testikel sind erbsen- bis bohnengroß, bäufig sehr hoch gelagert, so daß Andeutung von Kryptorchismus vorhanden ist. Auffälligerweise ist aber der Nebenhoden relativ gut entwickelt. Die Vesiculae seminales sind klein, zeigen in dem von 
uns mikroskopisch untersuchten Falle jene Verhältnisse, die wir beim Kastraten beschrieben haben.

Ähnlich verhält sich auch die Prostata; auch sie ist auffällig klein, unscharf gegen die Umgebung abgegrenzt, drïsenarm.

Das weibliche Genitale zeigt infantile Verhältnisse, der Uterus ist klein, kaum tastbar, ebenso die Ovarien.

Daß beim menschlichen Kastraten eine Vergrößerung der Hypophysis auftritt, haben wir seinerzeit nachgewiesen. Seither ist dieses Verhalten von INTAKa KoN an einem größeren Materiale bestätigt worden. Er fand die Hypophysen von Kastraten im Vergleich mit dem durchschnittlichen Gewicht nach Schoenemans um 1 bis $5 \mathrm{~g}$ schwerer. Auch sind sie in allen Durchmessern um einige Millimeter größer (Hypertrophie der chromophilen Zellen). Material: sechs wegen Carcinom oder Kystom kastrierte Frauen und ein wegen Tuberkulose kastrierter Mann.

Gleichzeitig haben wir auf die Vergrößerung der Hypophyse in der Gravidität hingewiesen, worauf schon vorher CoMTE u. a. aufmerksam gemacht hatten. In der Folge haben Erdheim und Stumme diese Frage ausfürlich bearbeitet und diese Tatsache bestätigt. Nenn demnach Funktionsänderungen oder Unterfunktionen des Ovariums während der Gravidität Veränderungen der Hypophyse, und zwar Vergrößerung dieses Organs bedingen, so macht diese Tatsache die Annahme wahrscheinlich, daß auch bei der genitalen Hypoplasie, beim Eunuchoid, hypophysäre Veränderungen, zumindestens funktioneller Natur, vorhanden sind.

In sämtlichen von uns beobachteten Fällen von Eunuchoiden konnte eine Vergrößernng der Hypophysis im Röntgenogramme nicht nachgewiesen werden, in dem obduzierten Falle war die Hypophyse makroskopisch nicht vergrößert, das Material für eine mikroskopische Untersuchung nicht mehr geeignet. Wir werden unten auf die bestehende Wechselbeziehung zwischen Genitaldrïsen und Hypophyse noch zurückkommen.

Nachdem wir im Vorhergehenden unsre eigne Kasuistik und die sich daraus ergebenden allgemeinen Gesichtspunkte beziuglich der Typen festgelegt haben, wollen wir nunmehr einige Angaben ans der Literatur heranziehen und diese kritisch sichten.

Diese Literaturangaben machen auf Vollständigkeit keinesfalls Anspruch, sondern sind wegen ihres illustrativen Wertes hier eingeschaltet. Wir können auf eine lückenlose Wiedergabe der Literatur 
um so eher verzichten, als der Eunuchoidismus keine Rarität, sondern eine häufige typische Anomalie darstellt.

Die für die hier aufgeworfenen Fragen wichtigen literarischen Daten werden im folgenden nach bestimmten Gesichtspunkten geordnet angeführt.

Wie schon einleitend bei der Besprechung der Nomenklatur hervorgehoben wurde, herrscht über die in Betracht kommenden Symptome im allgemeinen, bezüglich der sie auslösenden ätiologischen Momente im speziellen keine Übereinstimmung.

Was nun zunïchst den eunuchoiden Typus anlangt, so verweisen wir auf die Beschreibung eines 21jährigen Menschen durch Josef Griffith (1894), welcher für diese Fälle die Bezeichnung Eunuchoid gebraucht. Er beschreibt die Testikel, die Prostata und die Vesiculae seminales der betreffenden Leiche. Die Testikel sind klein (18 mm lang, $10 \mathrm{~mm}$ breit), von normaler Form, die Epididymis ist relativ groß, die Prostata ist klein, hart, mit spärlichen Drisenelementen, die Vesiculae seminales sind gleichfalls wenig entwickelt.

Henry Meige verweist in seiner Publikation L'infantilisme, le féminisme et les hermaphrodites antiques (1895) anf den von REICHLIN mitgeteilten Fall, einen 21 jährigen Mann betreffend, $178 \mathrm{~cm} \mathrm{lang,}$ $114 \mathrm{~kg}$ schwer, mit einer Kastratenstimme. Der Mann besitzt einen sehr kleinen Penis, ebensolche Testikel, keine Schamhaare, fublt keine Libido. Er war angeblich bis zum 13. Lebensjahre normal, begann um diese Zeit stark zu wachsen und fett zu werden.

SaINTON beschreibt einen hierhergehörigen Fall, ibber welchen er folgende charakteristische Beschreibung liefert: notre malade a la grande taille des eunuques $(1,72)$, il en a la longeur des membres disproportionés avec le corps, il en a l'absence de dévelopement du système pileux et le facies«. Sarnton gibt auch eine Familiengeschichte des betreffenden Kranken, aus welcher hervorgeht, daß unter den fünf Geschwistern des Patienten sich noch zwei eunuchoide Brüder befinden, ebenso soll ein Onkel des Patienten ein Eunuchoid gewesen sein. Auch der Großvater soll ein Eunuchoid gewesen sein (?).

Pirsche (1902) zitiert den Fall Papillault (1899) und bringt drei eigene hierhergehörige Beobachtungen bei.

Zweifelhaft bezüglich seiner Zugehörigkeit erscheint ein von BRISSAUd und MEIGE mitgeteilter Fall (1904), einen 30 Jahre alten Mann 
betreffend, mit bartlosem Gesicht, schwacher Behaarung an den Pubes und in den Axillae. Hoden bohnengroB, Penis der eines 15jährigen Knaben. Ossifikation vollendet. Die Sella turcica erschien groß. Die Autoren schildern den Patienten als »un petit géant $(1,85)$, un grand infantile, aussi un petit acromégaliquek.

Es scheint sich hier also um eine im Entstehen begriffene Akromegalie gehandelt zu haben.

Ausführlichere Berücksichtigung verdient ein von ReDLICH publizierter Fall von »Gigantismus infantilis « (1906).

31jähriger Mann, 1821/2 cm lang, $86 \mathrm{~cm}$ Sitzhöhe, 1901/2 cm Spannweite. Bis zum 24. Jahre gewachsen, seither nicht mehr. Nach Angabe seiner Schwestern war er bis zum 20. Jahre von normaler Größe, wurde dann lungenkrank und soll während seiner Erkrankung stark gewachsen sein. Hat niemals sexuellen Verkehr gehabt.

Ausgesprochener Schwachsinn. Gesicht völlig bartlos, nur ganz feine Lanugohaare. Gesichtshaut mager, stark faltbar, sehr gerunzelt, mit zahllosen Fältchen um die Augen und den Mund, dadurch von senilem Ausseben. Stimme auffällig hoch, knabenhaft. In den Achselhöhlen reichlich Haare, sonst am Rumpf und an den Extremitäten haarlos. Penis sehr kurz, an der Wurzel desselben findet sich ein schmaler Kranz hellblonder Haare.

Links ein haselnußgroßer, rechts ein etwas größerer Hoden zu fuhlen.

Die Röntgenuntersuchung ergibt ein mehr oder minder vollständiges Offenbleiben der Epiphysenfugen an den oberen und den unteren Extremitäten, nur am Ellbogengelenke sind die Epiphysenfugen verknöchert.

Sella tureiea normal.

-Es findet sich also das Bild eines gigantisch angelegten Skelets von schwächlichem inneren Aufbau, mit relativ unentwickelten Epiphysen und einem Ossifikationszustande, der einem Alter von 15-16 Jahren entspricht. *

RedLICH identifiziert diese Beobachtung mit dem Type infantile du gigantisme (Brissaud), erwähnt den Fall von Brissaud und Meige, sowie jene von Launors und Roy (Nouv. Ic. 15).

Die zitierte Arbeit von LaUnoIs und RoY betrifft den »grand Charles«, also einen Fall von Akromegalie. „Toutefois ce type de gigantisme infantile n'est peut-être pas irréductible, dans le temps 
au type de gigantisme acromégalique, l'hypertrophie hypophysaire causale étant commune à tous les deux."

Wir haben bereits auseinandergesetzt, aus welchen Grïnden wir für die in Rede stehende Krankheitsform die Partialbezeichnung Infantilismus ablehnen müssen; der Ausdruck Gigantismus ist gleichfalls nicht gerechtfertigt, da beim Eunuchoid ebenso wie beim Eunuchen nur ein Hinauswachsen tuber die für den betreffenden Volksstamm durchschnittliche Körperlänge vorliegt.

Es kann daher die wirklich erreichte Körperlänge kein Kriterium für den * Gigantismus* abgeben.

Daß in solchen Fällen die genitale Hypoplasie das Offenbleiben der Epiphysenfagen und damit die Möglichkeit des gesteigerten Längenwachstums bedingt, haben wir wiederholt hervorgehoben. Daß aber, wie Redlich mit Brissaud und Meige meint, der Anreiz zu dem gesteigerten Wachstum von der Hypophyse ausgeht, ist vorderhand nicht erwiesen. Die Annahme Reduichs, daß in seinem Falle, in welchem keine hypophysäre Veränderung nachweisbar war, der Anreiz für das vermehrte Längenwachstum in der familiären Disposition zu vermehrter Körpergröße gelegen ist, müssen wir zurückweisen, da wohl die familiäre Disposition die Größe des normalen Individuums bedingen kann, nicht aber den $\gg$ Anreiz zu vermehrtem Längenwachstum* nach Ausfall der Keimdrïsenfunktion.

DuckworTH beschreibt die Leiche eines 87 jährigen $»$ Eunuchoiden «, dessen größte Länge 177,7 cm, dessen Spannweite 179,2 cm beträgt. Derselbe hat femininen Typus, ist sehr fett, die Regio suprapubica besonders fettreich. Schambaare sehr spärlich. Penis und Skrotum sehr klein. Die beiden Testikel sind wenig entwickelt, die Vesiculae seminales fehlen angeblich vollständig. Die Prostata ist »nicht vergrößert $\ll$. Die mikroskopische Untersuchung der Testikel ergibt, daß die Canaliculi nur bindegewebige Stränge ohne Lumen darstellen, während die Epididymis aus dickwandigen, mit sehr feinem Lumen versehenen Kanälchen besteht. An der Prostata überwiegt hauptsächlich das fibröse Gewebe, da die Drübensubstanz sehr spärlich ausgebildet ist.

Hierher gehört auch der Fall Eicienne, Jeandelize and Richon, einen 55jährigen Mann betreffend, der $174 \mathrm{~cm}$ lang ist und an welchem die Verlängerung der unteren Extremitäten, die Bartlosigkeit und die hohe, schrille Stimme auffällt.

Das Individuum starb an einer Apoplexie und über den Sektionsbefund berichtet außer den erwähnten Autoren auch Christiax 
Champy, der eine histologische Untersuchung des Genitales vornahm.

Es handelt sich um ein vollständig bartloses Individuum mit zarter Haut. Der Kehlkopf ist klein, das Becken verbreitert. Die Epiphysenfugen sind unvollständig geschlossen, es bestehen an zwei Stellen Frakturen durch Lösung der Epiphysen.

Die äußeren Genitalien entsprechen in ibrer Entwickiung denen eines Knaben von 4-6 Jahren, der Penis miBt (am Lebenden) $4 \mathrm{~cm}$; auf einem Transversalschnitt zeigen die Corpora cavernosa einen Durchmesser von $1 \mathrm{~cm}$. Prostata etwa nußgroß, Samenblasen und das linke Vas deferens konnten nicht aufgefunden werden. Der linke Testikel findet sich im Hodensack, ist $2 \frac{1}{2} \mathrm{~cm}$ lang, $1 \frac{1 / 2}{\mathrm{~cm}}$ breit, wiegt drei Gramm. Der rechte Hoden liegt am äußeren Leistenring, seine Dimensionen entsprechen denen des linken, ebenso das Gewicht.

Mikroskopisch besteht der Hoden vorwiegend aus Bindegewebe, in welchem einzelne spärliche Inseln von Samenkanälchen zu sehen sind. Es finden sich keine Zwischenzellen.

Die Milz ist sehr klein (Gewicht $25 \mathrm{~g}$ ), die Thyreoidea hat normales Gewicht (29 g). Nebennieren normal.

Die Beobachtung von Thibierge und Gastinel betrifft einen 52 jährigen Mann, der $188 \mathrm{~cm}$ mißt, bei einem Körpergewicht von $86 \mathrm{~kg}$. Vom Trochanter bis zur Planta pedis mißt er $104 \mathrm{~cm}$. Er ist bartlos, hat wenig Achsel- und Schamhaare. Penis angeblich annähernd normal, Hoden rudimentär; Eunuchenstimme.

Die Autoren bezeichnen diesen Fall als , Gigantisme avec infantilisme $<$.

Weniger ergiebig ist die Literatur an Beobachtungen, welche die Kombination von Genitalhypoplasie mit excessivem Fettansatz ohne nachweisbare Hypophysenveränderung aufweisen, obwohl dahingehörige Individuen nach unsrer Erfahrung nicht so selten anzutreffen sind. Das hat darin seinen Grund, daß bei fetten Personen kindlichen und jurenilen Alters, welche ein Hauptkontingent der hierhergehörigen Fälle darstellen, die Untersuchung des Genitales unterlassen wird, oder die in solchen Fällen angetroffene genitale Hypoplasie mit dem Fettwuchs nicht in Beziehung gebracht wurde. Schließlich werden auch die veranlassenden Momente für den Fettwuchs in anderweitigen Veränderungen (andre Drüsen mit innerer Sekretion, Stoffwechselstörungen) gesucht.

KISCH betrachtet die hereditäre, in sehr jugendlichem Alter schon 
zum Ausdruck gelangte Fettsucht als einen eigentümlichen, nutritiven Ausdruck von Degeneration.

- Die erwachsenen Individuen dieser Kategorien (sc. der hereditär hochgradig lipomatösen männlichen Individuen) zeigen in der Körperkonfiguration einen mehr weiblichen Typus, der sich in den gebogenen Linien der gewölbten Schultern, in dem Hervortreten des Brustkorbes, in der Rundung der Hüften und Extremitäten bekundet. Das volle und runde Gesicht besitzt sehr spärliche Barthaare, oder diese fehlen meist vollständig. Die Mammae sind vollkommen weiblich, von halbkugelförmiger Gestalt bei jugendlichen, oder hängend bei älteren Individuen. Das männliche Genitale kann dabei normal oder auffallend mangelhaft entwickelt sein. Pubes spärlich oder gänzlich fehlend, der Penis zuweilen von absolut minimaler Größe, zuweilen aber nur durch die Fettwacherung zurückgedrängt, das letztere, indem die Fettzunahme des Panniculus adiposus der Bauchdecken und des Mons veneris den stets fettlos bleibenden Penis derart umschließt, daß dieser ganz zu verschwinden scheint, während die Fettentwicklung am Perineum und an den Oberschenkeln das gleichfalls des Fettes ermangelnde Skrotum nicht zur Geltung kommen läßt. Die Hoden sind oft wesentlich verkleinert oder es ist Kryptorchismus vorhanden. Die Stimme häufig ohne den sonoren männlichen Klang, oft an die der Kastraten erinnernd.

Neben diesem Feminismus bieten solche hereditär-lipomatöse Individuen nicht selten noch andre Degenerationszeichen: ein abnorm großes Körperwachstum in die Länge, Gigantismus oder Deformation der vergrößerten Ohrmuscheln, degenerierte Form der Kiefer, abnorme Entwicklung der Zähne.«

Wenn wir auch die musterhafte Beschreibung, die Kisch für diese Fälle gibt, in ihrer Gänze akzeptieren können, so müssen wir dennoch bemerken, daß der ursächliche Zusammenhang zwischen den körperiichen Veränderungen solcher Individuen und dem Zustande der Keimdrüsen von $\mathrm{K}_{\mathrm{ISCH}}$ nicht genügend betont worden ist. $\mathrm{Ob}$ die Ursache der Unterentwicklung des Genitales in einer allgemeinen Degeneration zu suchen ist, kann vorderhand nicht entschieden werden. Bemerkt sei nur, daß wir eine Reihe solcher Individuen ohne die geringsten somatischen Degenerationsmerkmale gesehen haben, weiter, daß in manchen Fällen deren Zustand ein passagerer ist und die Besserung mit der Entwicklung der Keimdrise zur Norm eintritt. Bezüglich der Heredität ist zu berücksichtigen, daß die eunuchoiden fettwüchsigen Personen in der Regel steril sind. 
EBSTEIN erwähnt bei einer zusammenfassenden Besprechung der Fettleibigkeit (Deutsche Klinik) die Kinder mit monströser Fettentwicklung, wie solche gelegentlich in Schaubuden zu sehen sind.

»Was ich davon gesehen habe, läßt mich annehmen, daß es sich in derartigen Fällen mehr um eine allgemeine excessive Körperentwicklung, um eine Art Riesenwuchs, als lediglich um eine reine Lipomatosis universalis handelt ...

Jedenfalls ist dieser als Polypionia infantum beschriebene Zustand, welcher wenigstens in sehr vielen Fällen mit der von SchösLeIN geschilderten sog. Chlororis gigantea zusammenfallen dürte, ein recht seltener. «

Den Zusammenhang der weiblichen Keimdrüsenfunktion mit excessiver Fettentwicklung behandelt in ausfuhrlicher Weise die Arbeit von BeRкowitch, welche allerdings die Fettentwicklung bei genitaler Hypoplasie nicht berüicksichtigt.

BERKowitch unterscheidet folgende Formen der $\gg$ obésité d'origine génitale beim weiblichen Geschlechte:

a) l'obésité de la puberté,

b) l'obésité post-nuptiale,

c) l'obésité maternelle,

d) l'obésité de la menopause,

e) l'obésité des ovariotomisées.

Bezüglich der unter e) eingereihten Fälle vgl. auch Alirerthum, ^Die Folgeznstände nach Kastration und die sekundären Geschlechtscharaktere. * Beitr. z. Geb. u. Gynäk. 1899. „Es ist nicht zu leugnen, daß sich bei einem großen Teil der kastrierten Frauen, ebenso wie im Klimakterium, Neigung zum Fettwerden bemerkbar macht. Eine sehr bedeutende Zunahme der Körperfülle konstatierten wir überhaupt nur in $29,5 \%$, also seltener als GlaEvECKE, der eine beträchtliche Zunahme des Körpergewichts in 57,5\%; des Fettansatzes in $42,5 \%$ gefunden hatte.*

Aus der letzten Zeit stammen die von KRÜCKMANN vorgestellten Fälle, zwei Geschwister betreffend, einen Jungen von 15 Jahren, ein Mädchen von 9 Jahren. Beide zeigen Opticusatrophie mit konzentrischer Gesichtsfeldeinschränkung. Der Junge wiegt 168 Pfund, das Mädchen 100 Pfund.

Hypophysenerkrankung nicht nachweisbar, Gegend der Sella normal.

Wie schon aus dieser kurzen Wiedergabe des klinischen Befundes erhellt, bedürfen diese Fälle noch dringend der Ergänzung. 
Bevor wir an die Besprechung der sog. hypophysären Fettsucht gehen, wollen wir noch auf jene Fälle hinweisen, in welchen Gehirnprozesse ohne erweisbare Veränderungen der Hypophyse mit Fettsucht und Veränderungen des Genitales in Zusammenhang gebracht wurden.

Zunächst ist eine Reihe von Fällen hier anzuführen, in welchen bei Hirntumoren der verschiedensten Lokalisation Menstruationsstörungen auftreten bzw. ein Frühsymptom des Hirnprozesses darstellen.

Hierher gehören z. B. der Fall Bayerthal (Stirnhirntumor), die Angaben von Axenfeld, daß bei basalen Hirntumoren Menstruationsstörungen vorkommen, » vermutlich durch Vermittlung der Hypophyse «. Weiter die Fälle von MüLLER, bei welchen bei Tumoren im Occipitallappen und im Kleinhirn Amenorrhoe, mit Fettsucht gepaart, beobachtet wurde, Breguan, Abelsdorf, Yamaguchi u. a.

Ferner sind hier die Fälle anzureihen, bei welchen die betreffenden Tumoren wohl in der Nähe der Hypophysis sitzen, ohne daß dieselbe nachweisbar beteiligt ist. So die Fälle von ZAK, BERGERErdhem (Fall II), Bartels.

Zak: Typisches Endotheliom der Dura mater mit reichlicher Psammombildung. Hypophyse und Infundibulum ergeben histologisch einen vollkommen normalen Befund.

Berger-Erdheim: An der Basis des Gehirns findet sich hinter dem Chiasma ein Tumor, der gegen die Hypophyse zu prominiert und von dessen unterem Umfang, wenn auch nicht gerade an der tiefsten Stelle, der Hypophysenstiel abgeht. Die Hypophyse ist dureh den darüber liegenden Tumor anseheinend komprimiert und abgeplattet, sonst makroskopisch (und mikroskopisch, ERDHEIM) ohne Veränderungen.

BARTELS: Hühnereigroße Plattenepithelgeschwulst ïber der Hypophyse.

Beobachtungen dieser Art wurden für einzelne Autoren der AnlaB eine gesetzmäßige Wechselbeziehung zwischen Hypophyse and Genitale iiberhanpt in Abrede zu stellen. So ERdherm, der die Meinung ausspricht, daß die bei Hypophysentumoren ohne und mit Akromegalie vorkommende Adipositas höchstwahrscheinlich nicht auf die Hypophyse zuriuckzuführen sei, sondern darauf, daß in solchen Fällen eine uns unbekannte Stelle der Hirnbasis durch den Tumor beeinflußt wird.

Auch Bartels kommt zu dem Schlusse, daß ein gesetzmäßiger Einfluß der Hypophyse anf das Genitale fehlt und sich aus der menschlichen Pathologie der Beweis einer Beziehung zwischen beiden nicht erbringen lasse.

Marburg meint, daß es sich bei Kleinhirnaffektionen mit Adipositas nicht um die funktionelle Schädigung der Hypophyse durch 
einen eventuellen Hydrops des dritten Ventrikels handle, welcher Anlaß zur Adipositas gebe, daß bei den Hypophysenganggeschwülsten nicht die Läsion irgendeines hypothetischen Centrums letztere hervorbringe, sondern daß das gemeinsame ätiologische Moment dieser in der Art des Tumors gelegen sei, der sich offenbar aus einem ganz bestimmten Gewebe zusammensetzen muß. Es hat den Anschein, daß gewisse embryonale, dem Ektoderm nahestehende Gewebe im besonderen diese Eigenschaft besitzen, woraus sich vielleicht auch der Umstand erklärt, daß gerade die Infundibularregion nnd das Kleinhirn Sitz solcher Affektionen ist. Nach dieser Auffassung wäre die Adipositas bei Hirntumoren ein Symptom, das topisch erst in zweiter Linie zu verwenden ist, das in erster Linie darauf hinweist, daBes sich um eine bestimmte Geschwulstart handelt.

In einer späteren Arbeit (1909) hat Marburg selbst diesen Standpunkt als irrig bezeichnet.

Wir erwähnen schließlich, daß auch bei Erkrankungen der Zirbeldrüse Fettsucht zur Beobachtung gelangt ist, gewöhnlich vergesellschaftet mit Frühentwicklung des Genitalapparates. [Literatur: MARbUrG, v. Frankl-Hochwart.]

Während in den oben angeführten Beobachtungen die Möglichkeit immerhin offen bleiben könnte, daß die Hypophysis, wenn auch nur mittelbar, funktionell gestört, also an dem Zustandekommen des Symptomenbildes beteiligt ist, gibt es eine stattliche Reihe von Fällen, in welchen die Wechselbeziehung zwischen Hypophyse und Genitale einwandfrei nachgewiesen werden kann. Zur Stütze dieser Wechselbeziehung kann auch eine Anzahl von experimentellen Tatsachen angeführt werden. Auf die Gefahr hin schon früher Gesagtes hier zu wiederholen, sollen die in dieser Richtung vorhandenen Tatsachen hier aufgezählt werden.

Nach der Kastration hypertrophiert die Hypophyse (Ficheras Experimente; Crmoroni, TandLER und Grosz); Hypophysenvergrößerung in der Schwangerschaft (Conte, Guerrini, Launois, Thaon, Cagnetto, Tandler und Grosz, Erdheim und Stummej.

Hypophysenextirpation bei Tieren führt zu regressiven Veränderungen am Genitale und Fettansatz (Cushing, Aschner). Implantation von Hypophysen bei Ratten bewirkt Fettansatz (A. ExNer).

Anderseits können nach Entfernung der erkrankten Hypophysis bei Akromegalie die genitalen Störungen versehwinden (HochenegG, v. Eiselsberg und v. Frankl-Hochwart). 
Die Wechselwirkung besteht also in doppelter Richtung, einmal rom Genitale auf die Hypophyse, das andre Mal in umgekehrter Richtung.

Hier sei mit Nachdruck bemerkt, $d a B$ sich die Wechselbeziehungen zwischen Hypophyse and Keimdruse nach unsern eignen Untersuchungen nur als solche zwischen Hypophysis und interstitieller Substanz charakterisieren lassen, daß die Veränderungen am generativen Anteile (Ductuli seminiferi, Follikel) sekundärer Natur sind oder wenigstens für die innersekretorische Funktion ohne Belang sind.

Wir bringen nunmehr die Krankengeschichten zweier Fälle von sog. hypophysärer Fettsucht. Der erste dieser Fälle ist seinerzeit (1901) von A. FröHLICH publiziert worden, wurde am 21. Juni 1907 von Hofrat v. Eiselsberg operiert, der zweite Fall wurde bereits von v. Eiselsberg und v. Frankl-Hochwart zur Kenntnis gebracht.

I.

R. D., 1899, 14 Jahre alt, Kopfschmerzen mit Erbrechen.

Augenhintergrund -normal.

Seit März 1899 rapide Zunahme des Körpergewichts.

Januar 1901 Herabsetzung der Sehkraft am linken Auge, schließlich Erblindung. Auch rechts Abnahme der Sehkraft.

Mai 1901 Gewicht $54 \mathrm{~kg}$.

Augenuntersuchung: genuine Atrophie des N. optic. links, rechts normal. Links Amaurose, rechts ${ }^{5 / 20}$. Temporale Hemianopsie rechts.

Die stärksten Anbäufungen von Fett in der Haut des Rumpfes, namentlich am Abdomen und in der Nähe des Genitales. Daselbst sind die Fettmassen so mächtig, daß sie sich rund um das Genitale stark vorwölben. Der Penis, der übrigens normal entwickelt ist, erscheint zwischen diesen Fettmassen derart eingelagert, daß das Genitale sich dem femininen Typus nähert.

Die Hoden sind in der Tiefe der Fettmassen palpabel und bieten infantile Verhältnisse dar.

In der Gegend der Mamillen finden sich gleichfalls nambafte Fettansammlungen.

Die Behaarung der Achselhöhlen fehlt, am Genitale finden sich nur vereinzelte Härchen. Die Haare am Schädel sind spröde und spärlich. Sie sollen seit Beginn der Erkrankung kontinuierlich aus- 
fallen. Soweit die seinerzeit von FröHuıCH veröffentlichte Krankengeschichte.

Sechs Jahre später bietet der Patient fast dieselben Symptome, nur ist noch eine weitere Abnahme seiner Sehkraft hinzugetreten. Er hat ein ganz infantiles Genitale, vollkommenen Mangel der Libido sexualis und der Erektionsfähigkeit. Scham- und Achselhaare fehlen. Röntgenologisch läßt sich Offenbleiben der Epiphysenfugen, weiter ein Hypophysentumor nachweisen.

Am 21. Juni 1907 wurde von Hofrat v. Erselsberg die Hypophysenexstirpation vorgenommen. Es fand sich eine große Cyste.

Im Juli 1908 zeigt der Patient folgendes Verhalten: die einige Zeit nach der Operation konstatierte Besserung des Sehvermögens hat angehalten. Kopfschmerz und Schwindel sind seit Monaten nicht mehr aufgetreten. In letzterer Zeit sollen angeblich Erektionen auftreten. An den Pubes und an den Axillae sehr spärliche Behaarung nachweisbar.

\section{II.}

K. W., 1881 geboren. Er wurde am 12. Juni 1906 von weiland Dr. BERGER im psychiatrischen Vereine mit der Diagnose Hypophysentumor vorgestellt. Er erschien anfangs Oktober 1907 mit ziemlich bedeutenden Beschwerden wieder. Die Mutter des Patienten ist fettleibig; sonst nichts Bemerkenswertes beztiglich der hereditären Verhältnisse. Der Patient hat keine Kinderkrankheiten durchgemacht; seit dem 18. Jahre besteht Obstipation und allgemeine Blässe. Er war stets fettleibig, will in den letzten Jahren an Gewicht noch zugenommen haben. Im Mai 1906 trat plötzlich eine Verschlechterung des Sehvermögens ein. Seit Herbst 1906 erbricht Patient leicht bei besonders heftigem Kopfschmerz.

Das eigentïmliche kindliche Anssehen des Kranken soll erst seit 1900 auffallend sein. Seit längerer Zeit besteht Ergrauen der Haare. Erektionen traten vom 10.-15. Lebensjahre des Patienten auf, seither nicht mehr. Ebenso fehlen seit dieser Zeit die Libido sexualis und Pollutionen.

Status präsens: Patient ist auffallend klein, $161 \mathrm{~cm}$. Die Haut auffallend weich und zart. Keine Andeutung von Bart-, Achsel- und Schamhaaren, der ganze Körper wie leicht gedunsen, ziemlich bedeutender Grad von Fettleibigkeit. Über der Peniswurzel reichlich Fettentwicklung, erinnernd an den Mons veneris einer Frau. Hoden deutlich vorhanden, jedoch klein. Schilddrüse normal. Die Knochen 
erscheinen normal, die radiologische Untersuchung ergab einen typischen Befund, Keilbeinkörper, Sattellehne, Processus clinoidei antici vollkommen fehlend.

Operation am 20. Dezember 1907 (Hofrat v. EiselsberG). Die Untersuchung der Geschwulst ergibt ein Angiosarcom.

Wir haben seinerzeit in unsern "Untersuchungen an Skopzen" die Frage offen gelassen, $\nsim 0 b$ in der Pathogenese der Akromegalie die Hypophysenerkrankung das Primäre, die Keimdrisenerkrankung das Sekundäre sei, oder ob beide koordiniert sind, oder endlich, $o b$ die Genitalerkrankung die Hypophysenveränderung auslöst «. Während aber seither durch die Operationsresultate der Chirurgen (HochenegG), durch die epxerimentellen Ergebnisse der jüngsten Zeit (Cushing, ASCHNER) diese Frage dahin zu beantworten ist, daß hier wohl die Veränderungen der Hypophyse das auslösende Moment für die Genitalveränderung darstellen, kann sie für die sog. hypophysäre Fettsucht noch als eine offene gelten.

A. FröHLICH gebührt das Verdienst, das klinische Bild der hypophysären Fettsucht zuerst erkannt und scharf umschrieben zu haben, seine Beobachtung betrifft den Fall D., den wir im vorstehenden weitergeführt haben. Das Schwergewicht der von FröнLich vorgebrachten klinischen Argumentation ruht in dem Nachweise, daß die Veränderung der Hypophyse die Fettzunahme bedinge. In zweite Linie stellt er die Anomalien der Behaarung, die Entwicklung der Brustdrüse und die eigentumliche "an das weibliche Genitale erinnernde Beschaffenheit der Schamgegend «. Die Unterentwicklung des Genitales wird von ihm im klinischen Status wohl erwähnt, ohne daß er die Frage aufwirft, ob und welche Bedeutung diesem Symptome zuzuerkennen ist. Schon Fröhlich beruft sich auf eine Reihe ähnlicher Beobachtungen (Mohr, Hippel, Glaeser, Wadsworth, WiLh, Boyces und Beadles, Pechkranz, Stewart), in der Folge ist eine ganze Reihe hierhergehöriger Fälle publiziert worden, welche in der Arbeit von ERDHEIM zusammengestellt und kritisch gesichtet wurde und in dem jungsten Referate von BorchardT eine Ergänzung erfahren hat.

Wie schon erwähnt, wendet sich ERDHeim gegen die Deutung FröHLICHs und meint, daß fur die Adipositas nicht die Hypophyse, sondern eine uns unbekannte Stelle der Hirnbasis verantwortlich zu machen sei. Er stitzt sich hierbei vornehmlich auf den Befund in einem eignen Falle und den Fall SeLke. 
Wir glauben auf eine detaillierte Wiedergabe der hierhergehörigen Publikationen verzichten zu können, da sie in der Symptomatologie eine weitgehende Gleichförmigkeit aufweisen.

Nur bezïglich der Pathogenese sei hier angefuhrt, daß einzelne Autoren sich der von FröHLICH geänßerten Anschauung vollkommen anschließen, andre (ScHüLLER) Hypohysenveränderung und Genitalatrophie als koordiniert, die Fettsucht als bedingt durch die Genitalatrophie betrachten, wieder andre den Zusammenhang zwischen Hypophysenerkrankung und Genitalveränderung als nicht erwiesen ansehen (BARTELS).

Wir haben schon erwähnt, daß die Erkenntnis des pathogenetischen Zusammenhangs zwischen Hypophysis, Genitale und Fettsucht auf Schwierigkeiten stößt und wollen diese hier in Ktirze auseinandersetzen. Eine der größten Schwierigkeiten ist darin gelegen, daß es fast niemals gelingt, die zeitliche Aufeinanderfolge der genitalen und der hypophysären Veränderungen festzulegen. In erster Linie fehlt hier die einwandfreie histologische Untersuchung der Hypophysis an Personen mit genitaler Hypoplasie und Fettsucht, weiter ein Maßstab fur die Bewertung quoad functionem der etwa erhobenen mikroskopischen Befunde. Anderseits steht auch die mikroskopische Untersuchung der Genitaldrüse bei Individuen mit sogenannter hypophysärer Fettsucht noch aus. Diesbezüglich liegt nur der von Nazari stammende Befund vor. Dieser Autor beschreibt bei einem 20 jährigen Individuum mit Hypophysentumor einen dem embryonalen entsprechenden Befund des Testikels.

Die vorhandenen Möglichkeiten in dem pathogenetischen $\mathrm{Zu}$ sammenhange sind nun folgende:

a) Primäre Erkrankung der Hypophysis, konsekutive Erkrankung des Genitales, dieser koordiniert (also ebenfalls direkt von der Hypophyse ausgehend) Fettwuchs.

In seltenen Fällen könnte nur eine der beiden Konsequenzen sich ausbilden, die andre ausbleiben.

b) Primäre Erkrankung der Hypophyse, konsekutive Erkrankung des Genitales, durch die letztere bedingt Fettwuchs.

c) Primäre Erkrankung der Keimdrüse, konsekutive Fettsucht und hypophysäre Veränderung koordiniert.

Auch hier könnte einer der beiden konsekutiven Prozesse ausbleiben.

d) Primäre Erkrankung der Keimdrüse, konsekutive Veränderung der Hypophyse, welche den Fettwuchs bedingt. 
Ob alle diese Möglichkeiten wirklich vorkommen, welche derselben die häufigsten sind, wird erst zu entscheiden sein, wenn eine verfeinerte klinische Untersuchung, eventuell gestützt durch biologische Experimente, die führende Rolle übernehmen kann.

Zum Schlusse möchten wir noch mit einigen Worten auf das Verhalten der Thymus beim Eunuchoidismus bzw. auf die Abgrenzbarkeit dieses Symptomkomplexes gegentiber jenem, der als Status thymicus, Status thymico-lymphaticus, hypoplastische Konstitution bezeichnet wird, eingehen. Beim kastrierten Tier bleibt als eine Manifestation der Unreife die Thymus länger bestehen als normal. (Henderson, Journ. of Phys. Vol. 31, Soli, Arch. it. de biol. 1907.) Es war nun von vornherein wahrscheinlich, daß auch bei der genitalen Unterentwicklung Thymuspersistenz vorkomme. Tatsächlich findet man auch am Röntgenogramme der Brustorgane solcher Individuen einen Schatten, der als Thymusschatten bezeichnet werden könnte, wenn auch nicht verhehlt werden soll, daß die Deutung dieses Schattens rielfach Schwierigkeiten begegnet. An Wahrscheinlichkeit gewinnt diese Auffassung durch den Umstand, daß wir bei der Sektion jugendlicher Personen mit genitaler Hypoplasie Thymuspersistenz nachweisen konnten.

Wir verdanken die betreffenden Präparate der Liebenswürdigkeit des Herrn Professor Kolisko.

In dem seinerzeit von A. Paltaur aufgestellten Symptomenkomplex des Status thymicus wurde auf die Unterentwicklung des Genitales keine Rücksicht genommen, wenigstens wurde sie nicht als zu dem Komplex gehörig beschrieben.

In neuerer Zeit hat BARTEL bei der *hypoplastischen Konstitution * die genitale Hypoplasie ebenfalls angeführt, Kyrle und Hermaxx konnten die morphologischen Eigentümlichkeiten der Hoden und Ovarien bei solchen Individuen studieren.

Nach BaRTEL zeigte das Genitale in solchen Fällen, namentlich bei weiblichen Personen, Hypoplasie, die sich schon durch Menstruationsanomalien verriet. Mangelhafte Crines, enge Vagina, Uterus infantilis, meist übermäßig große, oft ganz glatte Ovarien, häufig mit Cystenbildung bei älteren Frauen, waren die Kennzeichen beim Weibe, mangelhafte Crines und Kleinheit der äußeren Geschlechtsteile beim Manne.

E. Hermand berichtet uber 55 Fälle: 5 Fälle von Kindern $\left(5 \frac{1}{2}\right.$ bis 12 Jahre), 19 Fälle von Jugendlichen (14 bis 20 Jahre), 31 Fälle von geschlechtsreifen Frauen (21 bis 46 Jahre). 
- Die Ovarien sind in $580_{0}$ vergrößert und zeigen ein Längenmaß von 5,6 , ja selbst $8,5 \mathrm{~cm}$. Einerseits zeigen sie einen die normale Form des 0variums beibehaltenden Typus, nur sind die Maße nach allen drei Richtungen größer; anderseits zeigen sie den animalen Typus: langgestreckt und schmal.

Oberfläche glatt, Konsistenz vermehrt. Am Durchschnitt eine große Zahl von subcortical gelegenen eystischen Gebilden. Die Grüße dieser >Cysten schwankt zwischen Hirsekorn- bis Kirschengröße.

Mikroskopisch zeigt es sich, daß die Vergrößerung des Ovariums auf einer Zunahme der bindegewebigen Anteile desselben beruht, insbesondere ist es die Albuginea, die daran ganz besonders teilnimmt. Das Bindegewebe ist häufig zellarm, faserig, ja partienweise geradezu schwielig.

Primärfollikel normal geformt, jedoch in geringerer Zahl. Die cystischen Gebilde erweisen sich als dilatierte Graarsehe Follikel. Viele Follikelatresien, spärliche Corpora lutea, hingegen reichlich Corpora fibrosa.

Die Verdickung der Rinde verhindert das Platzen der Follikel, infolgedessen Follikeldilatation, hierauf Absterben des Eies und Atresie.

Die Gefäßstämme im Hylus sehr häufig verdickt, die Wand häufig hyalin degeneriert, daneben vollständige Obliteration.

Die Uteri dieser Fälle meist hypoplastisch; Länge 4 bis $5 \mathrm{~cm}$, Wand dünn, Endometrium atrophisch. Dementsprechend in $20 \% 0$ unsrer Fälle vollständige Amenorrhüe; in $24 \%$ vielmonatliche Cessationen. In $38 \%$ ein- oder mehrmonatliche Graviditiat.

Hinweis auf die Ähnlichkeit des histologisehen Bildes mit der chronischen Oophoritis. Bei der chronischen Oophoritis stets eine Hypertrophie auch am sonstigen inneren Genitalapparat, während hier eine Hyperplasie der bindegewebigen Anteile des Eierstockes vorkommt und der Uterus meist hypoplastisch bleibt. Die prägnantesten Erscheinungen schon bei Kindern, die chronische Oophoritis mehr bei Erwachsenen.

Inwieweit diese Erscheinungen fiir den Status lymphaticus charakteristisch sind und ob sie durch ihn bedingt sind, ist derzeit noch nicht zu entscheiden.

KyrLE demonstriert vier histologische Präparate von menschlichen Hoden, die von Individuen mit ausgesprochenem Status hypoplasticus gewonnen wurden. Das eine Präparat stammt von einem 14jährigen Individuum, und ist in gewissen Punkten verschieden von den drei andern Präparaten, welche von älteren Personen hergenommen sind. Nan sieht bei diesem Hoden auf den ersten Blick, daß es sich um einen nicht entsprechend entwickelten Hoden handelt; die Hodenkanälchen sind sehr klein im Durchmesser, die Membrana propria ist ziemlich dick, das Hodenzwischengewebe, das Stroma, ist mächtig entwickelt, Hodenzwischenzellen sind vorhanden. Das Kanälchenepithel besteht aus fast nur einer einzigen Zellgattung, nirgends sind Ansätze einer Spermatogenese zu sehen. Das Bild dieser Hoden erinnert am meisten an Testikel vom Menschen knapp vor der Geburt und kurze Zeit post partum, nur daß das Zwischengewebe in dem vorliegenden Falle viel reichlicher entwickelt ist. Es kann gar keinem Zweifel unterliegen, daß hier eine schwere Entwicklungshemmung vorliegt. Mit diesem übereinstimmende Befunde konnten an mehreren untersuchten hypoplastischen Individuen der ersten Lebensjahre konstatiert werden. Bei den drei andern Präparaten von Hoden hypoplastischer Individuen - es handelt sich um Personen zwisehen 20 bis 30 Jahren - ist das Bild eigentlich ein ganz andres. Hier liegen voll entwickelte Hoden vor. die gewisse Veränderungen 
aufweisen; es sei nebenbei erwähnt, daß diese Hoden makroskopisch keine Veriinderungen aufweisen, von normalen, besonders an Größe, nicht unterschieden werden können, während z. B. der Hoden des 14 jährigen Knaben schon deutlich atrophisch aussah, viel kleiner war, als dies für ein 14jähriges Individuum zu erwarten ist. An diesen Hoden der Erwachsenen findet man eine deutliche Verdickung der Hodenkanälchengrundmembran, nebst einer stellenweise recht intensiv entwickelten Stromavermehrung; in das Stroma eingestreut finden sich Hodenzwischenzellen, zum Teil vereinzelt, zum Teil in Häufchen gelagert. Das Epithel der Kanälchen zeigt kein einheitliches Verhalten in den verschiedenen Fällen, indem in der einen Reihe von Fällen die Spermatogenese nicht gestört erschien, während in andern Fällen dieselbe sichtlich geschädigt war. Der Grad der Bindegewebsvermehrang ist gleichfalls nicht in allen untersuchten Fällen derselbe. Aber in allen zwanzig nntersuchten Fällen konnten Veränderungen dieser Art nachgewiesen werden."

Wir selbst haben seinerzeit auf die bestehende Wechselwirkung zwischen Keimdrüse und Thymus hingewiesen.

Die Abgrenzung des Eunuchoids gegen den Status thymolymphaticus (hypoplastische Konstitution nach BARTEL) der Autoren zu geben, sind wir vorläufig außerstande, schon deshalb, weil von seiten jener Autoren, welche sich mit diesem Symptomenkomplex beschäftigt haben, die Frage betreffend den ursächlichen Zusammenhang zwischen genitaler Hypoplasie und Thymuspersistenz zu wenig gewürdigt wurde. Erst eine Untersuchung des Statns thymo-lymphaticus, bei welcher der Genitalzustand nicht Befund, sondern Ausgangspunkt bildet, kann in dieser Frage Klärung bringen.

\section{Literaturverzeichnis.}

Abelsdorf, Klin. MIonatsblätter für Augenheilkunde. 41. 1903.

Alterthum, Ernst, Die Folgezustände nach Kastration und die sekundären

Geschlechtscharaktere. Beitr. z. Geb. u. Gynäk. Bd. 2. 1899.

Ammon, Otтo, Linfantilisme et le féminisme an conseil de revision. L'anthropologie. 1896.

Aubry, Jeandelize, Richon. A propros d un type d'infantile à longs membres avec persistance des cartilages epiphys. C. r. soc. biol. 1906.

Axesfeld, Neurol. Centralblatt. 1903. S. 608.

Babinski, Rev. neurol. 1900.

BARTeL, Über die hypoplastische Konstitution. Gesellschaft für innere Medizin

Wien. 1908. Wiener kl. W. 1908.

Bartels, MLartin, Zeitschrift für Augenheilkunde. 1906.

Bayerthal. Neurol. Centralblatt. 1903. S. 572.

Berger, A., Ein Fall von Tumor der Hypophysengegend. Zeitschr. f. klin. Med. Bd. 54. 1904. 
Berger, Beitrag zur Kastration und deren Folgezustände. Dissertation. Greifswald 1901.

Berkowitch, De l'obésité d'origine génitale chez la femme. Thèse Paris. 1908.

Bonchardt, Ergebnisse der inneren Medizin und Kinderheilkunde. Bd. 3.

Boyces and Beadles, A further contribution to the study of the hypophysis cerebri. Journ. of path. and bact. 1893.

Bregman und Steinhaus, Virchows Archiv. 188. 1907.

BrissaUd, L'infantilisme vrai. Nouv. Icon. Vol. 20. 1907.

Brissaud et Mleige, Type infantile du gigantisme. Nouv. Icon. 1904. Vol. 17.

Bychowski, Zar Diagnose and Therapie der Hypophysisgeschwïlste. D. med. Wochenschr. 1909. Nr. 36.

Cagnetto, Virchows Archiv. 176; 187.

Chaspy, CHr, Étude histologique du testicule d'un homme, qui présentait les charactères d'un castrat. Compt. rend. soc. biol. Vol. 62.

Cmroroni, Lo speriment. 61. 1907. Arch. it. de biol. 48. 1908.

Claude et Govgerot, Sur linsuffisance simult. de plusieurs glandes à secret. int. C. r. biol. 1907.

Comrs, Thèse inaug. Lausanne 1898.

- Contribution a l'étude de l'hypophyse humaine et de ses relations avec le corps thyrèide. Zieglers Beiträge. 1899. Bd. 23.

Crzellitzer, Geschwulst in der Hypophysengegend mit ungewöhnlichen Sehstörungen. Berl. kl. Wochenschr. 1909. Nr. 20.

Gushing, H., Sexnal Infantilism with Optic Atrophy in cases of Tumor Affecting the Hypophysis Cerebri. Journ. of Nerv. and Ment. Disease. 1906.

— J. Americ. Med. Assoc. Chicago. Ref. M. M. W. No. 37. 1909. p. 1912.

Duckworth, Notes on the Anatomy of an Eunuchoid man. Journ. of Anat. and Phys. 41.

Dupré et Pagnez, Infantilisme dégénératif (Type Lorain) compliqué de dysthyroïdie pubérale (Type BrIssavd;. Nouv. Icon. 1902. p. 124.

v. Eiselsberg und v. FrankL-Hochwart, Über operative Behandlung der Tumoren der Hypophysisgegend. Neurol. Centralblatt. 1907.

— Ein nener Fall von Hypophysisoperation bei Degeneratio adiposogenitalis. Wiener klin. Wochenschr. 1908. Nr. 31.

Eisenlour, Virchows Archiv. Bd. 68.

Erdheim und Stumme, 37. Chirurgenkongreß. 1908.

_- — Über Schwangerschaftsveränderungen der Hypophyse. ZIEGLERs Beiträge. Bd. 46. 1909.

Erdhem, Zieglers Beiträge. Bd. 33.

Etienne, Jeandelize et Richox, Malformations organiques multiples chez un castrat naturel. Compt. rend. soc. biol. T. 62.

Exner, A., Beiträge zur Pathologie der Hypophyse. 81. Versammlung deutscher Naturforscher und Ärzte. Salzburg 1909.

Fichera, Gaetano, Sulla ipertrofia della ghiandola pituitaria consecutiva alla castrazione. Policlinico 1905.

Bull. R. Acad. Roma. XXI. Fasc. 3 e 4. 1905.

Finkelnburg, Deutsche Zeitschrift für Nervenheilkunde. Bd. 21.

r. Frankl-Hochwart, Die Diagnostik der Hypophysistumoren ohne Akromegalie. Wiener med. Wochenschr. 37. 1909. 
v. Frankl-Hochwart, Über Diagnose der Zirbeldrïsentumoren. Deutsche Zeitschrift f. Nervenheilkunde. Bd. 37.

Fröhlich, A., Ein Fall von Tumor der Hypophysis cerebri ohne Akromegalie. Wiener klin. Rundsch. 1901.

Fuchs, Alfred, Zur Frühdiagnose der Hypophysistumoren. Wiener klinische Wochenschrift. 1903.

— Verein für Psychiatrie und Neurologie in Wien, 14. März 1905. Wiener klin. Wochenschr. 1905. S. 367.

Garsier, Cryptorchidie chez l'homme adulte stérile avec conservation de la fonction diastématique. C. r. biol. 1909.

GLäSER, Virchows Archiv. 122. 1890.

GLAEvecke, Körperliche und geistige Veränderungen im weiblichen Körper nach künstlichem Verlust der Ovarien. Arch. f. Gynäk. 35. 1899.

GöTzL und ERDHEIM, J., Zur Kasuistik der trophischen Störungen bei Hirntumoren. Zeitschrift für Heilkunde. 1905.

Griffith, Joseph, The condition of the testes and prostata gland in eunuchoid persons. Journ. of Anat. Vol. 28. 1894.

Gross et Sexcert, Décollement épiphysaire chez un castrat naturel adulte. C. r. biol. 1905 .

Gruber, W., Über die kongenitale Anorchie beim Menschen. Medizin. Jahrbuch. 1868.

GuERrini, Arch. ital. de biol. 1905.

Henderson, On the relationship of the thymus to the sexual organs. The Journal of phys. Vol. 31. 1904.

Herrmanx, E., Demonstration von Ovarien beim Status lymphaticus beziehungsweise hypoplasticus. Centralblatt für Physiologie. Bd. 23. Nr. 8.

Hikmet et Regnault, Bulletin de la soc. d'anthropologie. 1901.

v. HipPes, Ein Beitrag zur Kasuistik der Hypophysistumoren. Virchows Archiv. 126. 1891.

HocheneGG, 37. Chirurgenkongreß, 1908.

Hudovernig et Popovits, Gigantisme précoce avec développement précoce des org. génitaux. Nouv. Icon. 1903. Bd. 16.

IngERManN, Diss. Bern. 1889.

KIscH, Über Feminismus männlicher lipomat. Individuen. Wiener med. Wochenschrift. 1905.

- Die Lipomatosis als Degenerationszeichen. Berliner klin. Wochenschrift. 1904.

KollaRits, Hypophysistumoren ohne Akromegalie. Deutsche Zeitschrift für Nervenheilkunde. Bd. 28. 1904.

Kon, Intaka, Hypophysenstudien. Zieglers Beiträge. Bd. 44. 1908.

KRÜCKMANN, Adipositas universalis bei zwei Geschwistern. Deutsche medizin. Wochenschr. 1908. S. 574.

KYrLE, Demonstration von Hoden beim Status lymphaticus beziehnngsweise hypoplasticus. Centralblatt für Physiologie. Bd. 23. Nr. 8.

Launors, Les cellules siderophiles chez la femme enceinte. Comptes rendus biol. 1903.

— Thèse de Paris. 1904.

- Castration et atrophie de la prostate. Assoc. franc. pour l'avancement des sciences. Congrès de Caen. 1894. 
Launois et Mulon, Les cellules cyanophiles chez la femme enceinte. Comptes rend. 1903.

Launors et Ror, Études biol. sur les géants. Paris 1904.

- Des relations qui existent entre l'état des glandes génit. mâles et le développement du squelette. Soc. de biol. 10 janvier 1903.

- - Gigantisme et castration. Revue intern. de médecine et chirurgie. Déc. 1902.

—— Gigantisme et infantilisme. Nouv. Icon. 15. 1902.

LAURENT, Leipzig 1896.

Lemos, Magalhaess, Infantilisme et dégénérescence psychique. Nouv. Icon. 1906. Bd. 19.

Lorter, Allongement des membres inf. chez un eunuque. Arch. danthrop. criminelle. Lyon 1896.

- Soc, de méd. de Lyon. 16 mars 1896.

Marburg, 0, Zur Kenntnis der normalen und pathologischen Anatomie der Zirbeldrüse. Arbeiten aus dem neurologischen Institut Wien. Bd. 17.

- Zur Frage der Adipositas universalis bei Hirntumoren. Wiener medizin. Wochenschr. 1907.

Matignon, J. J., Superstition Crime et Misère de Chine. Lyon 1899.

- Annales des malad. des org. génito-urin. 1896.

MAYER, Ein Beitrag zur Lehre von der Hypoplasie der Genitalien und vom Infantilismus auf Grund klinischer Beobachtungen. HEGAls Beiträge zur Gyn. u. Geb. Bd.12. 1907.

Meige, Henry, L'infantilisme, le féminisme et les hermaphrodites antiques. L'anthropologie. 1895.

- Sur le gigantisme. Arch. gén. de médecine. 1902.

Mingazzini, Gesellschaft deutscher Nervenärzte. Dresden 1907. Ref. Neurol. Centralblatt. 1907.

MöвIUs, P. J., Über die Wirkungen der Kastration. Halle 1903.

MoHr, Hypertrophie (markschwammartige Entartung?) der Hypophysis cerebri. Schmids Jahrb. 30. 1841.

Müller, Eduard, Über die Beeinflussung der Menstruation durch cerebrale Herderkrankungen. Neurol. Centralbl. 1,905.

Nazari, Il Policlinico. 1906.

- Ref. Centralblatt für allgemeine Pathologie. Bd. 19. 1908.

Noorden, K., y., Die Fettsucht. Nothnaget, Path. u. Therap. Bd. 7.

Parhon et Golstein, Les secretions internes. Paris 1909.

Parhon et Zalplacta, Sur un cas de gigantisme précoce avec polysarcie excessive. Nouv. Icon. 1907. Bd. 20.

Paton and Goodall, The journ. of phys. Vol. 31. 1904.

Pechkranz, Zur Kasuistik der Hypophysistumoren (Sarcoma angiomatodes hyp. cerebri). Neurol. Centralblatt. 18. 1899.

Pinsche, De l'influence de la castration sur le développement du squelette. Paris 1902.

PrtTard, La castration chez l'homme et les modifications qu'elle apporte. Comptes rendus de l'acad. des sciences. 8 juin 1903.

- Bulletin de la soc. des sciences de Bucarest. 1903.

Pittard, Eugène, La taille, le buste, le membre inf. chez les individus qu'ont subi la castration. Compt. rend. Ac. sc. T. 139. 
Poxcet, Lyon med. 1896.

RAth, Beiträge zur Symptomenlehre der Geschwiilste der Hypophysis cerebri. Arch. f. Ophthalm. Bd. 34. 1888.

Reduch, Ein Fall von Gigantismus inf. Wiener klin. Rundschau. 1896.

Richon et Jeandelize, Remarques sur la tête osseuse des lapins adultes castrés dans le jeune âge. C. r. biol. 190ó.

- Influence de la castration et de la résection du canal déférent sur le développement des org. génitaux ext. Comptes rend. hebd. de la soc. de biol. 1903.

_- Castration pratiquée chez le lapin. Comptes rend. biol. 1905.

- Influence de la castration et de l'ovariotomie totales sur le développement des org. génitaux ext. chez le jeune lapin. Comptes rend. soc. de biol. 1903. p. 1685 .

Richter und Loewy, Sexualfunktion und Stoffwechsel. Du Bors Archiv. Suppl. 1889.

Rieger, C., Die Kastration. Jena 1900.

Rosenhaupt, Heinrich, Ein Beitrag zur Klinik der Tumoren der Hypophysis. Berliner klin. Wochenschr. 1903.

Roy, Contribution à l'étude du gigantisme. Thèse de Paris, 25 février 1903.

Sainton, PaUl, Un cas d'eunuchisme familial. Nouv. Icon. XV. 1902.

Sellhem, Hugo, Kastration und Knochenwachstum. Beiträge z. Geb. u. Gyn. 1899.

Selke, Diss. Künigsberg. 1891.

SCHLOFFer, Wiener klin. Wochenschr. 1907.

SchüLler, Keimdrïsen und Nervensystem. Festschrift f. Orersteiner. Arbeiten aus d. neurolog. Institut der Wiener Lniversität. Bd. XV u. XVI. 1907.

_- Gesellschaft deutscher Nervenärzte. Dresden 1907. Ref. Neurol. Centralblatt. 1907.

Steinhaus, Virchowg Archiv. 188.

ThaOn, Thèse de Paris. 1907.

Thibierge et Gastinel, Un cas de gigantisme inf. Nouv. Icon. 1909.

Thom, Waldemar, Arch. f. mikr. Anat. Bd. 57.

WaDSWorth, Boston med. and surg. J. 1885.

White, Häusliches Leben und Sitten der Türken. Berlin 1845.

Wiehing, Beiträge zur Frage des allgemeinen Riesenwuchses. Deutsche med. Wochenschr. 1903.

Yamaguchi, Klinische Monatsblätter für Augenheilkunde. 1903. Beilagenheft. S. 180.

$\mathrm{Z}_{\mathrm{AK}}$, Über Hypophysistumoren. Wiener klin. Rundschau. 1904.

Zöllner, Tumor der Schädelbasis. Arch. f. Psych. 44. 1908. 
324 Julius Tandler a. Siegfr. Grosz, Über den Einfluß der Kastration usw. III.

\section{Erklärung der Abbildungen.}

Tafel VIII-X.

Fig. 1. Skelet des Eunuchoiden M. H. (Fall I).

Fig. 2. Prostata und Vesiculae seminales des Eunuchoiden M. H. Von hinten gesehen. Natürl. Gr.

$\operatorname{Pr}$ Prostata, Vs Vesicula seminalis, $D d$ Ductus deferens.

Fig. 3. Hoden und Nebenhoden des Eunuchoiden M. H.

Ep Epididymis, $T$ Testikel.

Fig. 4. Eunuchoid Adolf L. (Fall II).

Fig. 5. Eunuchoid Karl K. (Fall III).

Fig. 6. Eunuchoid Karl K. Genitalregion.

Fig. 7. Eunuchoid Heinrich Str. (Fall IV).

Fig. 8. Eunuchoid Josef Sch. (Fall V).

Fig. 9. Eunuchoid E. B. (Fall VII).

Fig. 10. Eunuchoid H. (Fall IX).

Fig. 11. Eunuchoid A. E.

Fig. 12. Eunuchoid St.

Fig. 13. Eunuchoid H.

Fig. 14. Fall D. nach Exstirpation der Hypophysis. 